Letter to the Editor

\title{
Reply - Letter to the Editor - Malnutrition: The kiss of grim reaper
}

Conducting a large, multicenter, prospective, randomized clinical trial poses a number of challenges. In particular, the successful completion of the trial is based on devising a protocol which could yield to clinically relevant answers yet no becoming so complex and sophisticated to prevent centers' participation and patients' enrollment. Consequently, the methodology of any trial reflects the clinical questions it tries to address.

The primary goal of the NOURISH study was to assess whether nutritional supplementation of malnourished, older, hospitalized patients led to improved clinical outcome [1]. Considering the large sample targeted, the authors assessed the presence of malnutrition using a robust and largely used nutritional assessment tool, SGA. It is agreed, as Sengul Aycicek et al. commented [2], that using imaging technique or measuring specific proteins' levels could have led to a more precise identification of the type of existing malnutrition. However, the focus of the trial is not if and how malnutrition improves following supplementation. Also, a complex procedure to assess nutritional status could have introduced interpretation biases, since circulating protein levels are influenced by the acute phase response, and patients enrolled were suffering of acute diseases, like pneumonia, or exacerbations of chronic diseases, like chronic obstructive pulmonary disease [1].

Sengul Aycicek et al. raises an important issue, i.e., whether the results obtained could have been at least in part secondary to a difference in physical activity levels between treated and placebo groups. A careful analysis of the manuscript reveals that no difference was observed between groups as far as a the Activities of Daily Living are considered [1]. Therefore, it is unlikely, although not certain, that physical activity levels in both groups were comparable throughout the study period. It is agreed that structured physical activity is key to enhance the anabolic properties of nutritional supplementation [3]. However, increased anabolic activity has been also demonstrated after nutritional supplementation during bedrest [4].

There is no perfect methodology for clinical trials, and studies could have been always done better. In fact, one of the most exciting aspects of clinical research is not only to inform clinical practice, but to allow to repeat, confirm and go beyond the current knowledge.

\section{Conflict of interest}

Dr. Laviano gave independent lectures at scientific and educational events sponsored by nutrition industries.

\section{References}

[1] Deutz NE, Matheson EM, Matarese LE, Luo M, Baggs GE, Nelson JL, et al. Readmission and mortality in malnourished, older, hospitalized adults treated with a specialized oral nutritional supplement: a randomized clinical trial. Clin Nutr 2016;35:18-26.

[2] Sengul Aycicek G, Arik G, Ulger Z. Malnutrition: the kiss of grim reaper. Clin Nutr 2016.

[3] Deutz NE, Bauer JM, Barazzoni R, Biolo G, Boirie Y, Bosy-Westphal A, et al. Protein intake and exercise for optimal muscle function with aging: recommendations from the ESPEN Expert Group. Clin Nutr 2014;33:929-36.

[4] English KL, Paddon-Jones D. Protecting muscle mass and function in older adults during bed rest. Curr Opin Clin Nutr Metab Care 2010;13:34-9.

\section{Alessandro Laviano* Department of Clinical Medicine, Sapienza University, Viale dell'Università 37, 00185 Rome, Italy \\ * Tel.: +39 0649973902; fax: +39064440806. E-mail address: alessandro.laviano@uniroma1.it.}

28 April 2016 